Materials science

\section{Making fuel for inertially confined fusion reactors}

\author{
from Robert $W$. Cahn
}

THE proceedings ${ }^{1}$ have recently been published of the Third Topical Meeting of Fusion Reactor Materials, held in September 1983. The very varied and exhaustive papers on tokamaks and their numerous materials problems include nothing whatever on the design and fabrication of the fuel micropellets needed for inertially confined fusion (ICF) reactors. Indeed, ICF is not mentioned anywhere. What accounts for this absence?

The previous meeting in this biennial series (held in August 1981) ${ }^{2}$, and a brief attempt to gaze into the future of fusion power, published in the same year ${ }^{3}$, likewise had no mention of this important topic. If, however, we go back to the First Topical Meeting ${ }^{4}$, held in January 1979, we find a group of nine papers concerned with ICF fuels, covering in considerable detail the position at that time concerning the design of the fuel microspheres, their fabrication and characterization, and the tricky problem of protecting the first wall of an ICF reactor from rapid series of miniexplosions. Why the near-total silence since then?

A very readable review paper on 'the special materials in laser fusion targets' has just been published ${ }^{5}$. The manuscript was received by the editor of Res Mechanica in April 1983, and the author states that he is presenting "the state of the technologies in making these materials, and problems encountered in their development, as of March 1980'. So it seems, possibly because of the connection of these technologies and problems with the military uses of lasers, that a security blackout descended after March 1980. I have seen a reference to a " 1980 Topical Conference on Inertial Confinement Fusion in San Diego" but no indication that the proceedings were published. Even with what we have, however - the 1979 papers and this 'postdated' $1980 / 1984$ paper - it is possible to see the quite extraordinary complexity of the problems and techniques involved. Hendrick's ${ }^{6}$ assertion that "pellet fabrication is not a trivial task as implied by some reports; it is also not the impossible task implied by others"' is to be noted.

ICF reactors are designed on the basis of the inertial confinement principle - a huge energy pulse imparted by laser or particle beam implodes a spherical shell of frozen deuterium-tritium mixture with a view to satisfying the Lawson criterion and setting off a series of microthermonuclear fusion explosions, the heat from which is absorbed and utilized to make power. In their review paper Farnum and Fries ${ }^{5}$ discuss the early stages of the development of a complex laser fusion target designed for maximum fusion yield when irradiated with $100 \mathrm{~kJ}$ of infrared light from a $\mathrm{CO}_{2}$ laser with $1 \mathrm{~ns}$ pulses. The essential parts consist of a glass microballoon coated internally with a layer of deuteriumtritium fuel and externally with a dense 'pusher' layer, the whole separated by plastic foam from an external dense metal pusher layer which is itself coated by an ablative plastic layer which creates the 'rocket' effect.

The difficulties arise from the extreme precision needed in the sphericity, thickness uniformity of layers, smoothness of surfaces (to within a few $\mathrm{nm}$ ) and uniformity of density, for instance of the plastic foam - and all this for microspheres usually well below $1 \mathrm{~mm}$ in external diameter. Microballoons can be bought commercially at $\$ 10^{-9}$ each, but their quality is hopelessly inadequate; the new paper outlines methods, starting either from liquid drops or from solid fragments in free fall, for making balloons with the requisite geometrical perfection. These thin shells can be filled with a gas mixture while in the gel state and then vitrified. (The method for turning the gas filling into a liquid spherical shell that is then frozen so rapidly that gravity has no time to distort it was described earlier ${ }^{7}$; this is really a kind of splat-quenching direct from the gas phase.) Problems connected with the buckling of the microballoons under gas pressure, and the effect of thin coatings on the buckling resistance, are discussed by Farnum and Fries.

The second part of their paper deals comprehensively with ways of making smooth uniform coatings of metals, polymers or a combination of these, by various forms of physical or chemical vapour deposition. These methods often depend for their efficacy on methods of levitating the spheres to be coated, either by systems of gas jets or even by whirling them around, during coating, in a form of synchrotron ${ }^{6}$. Remarkably perfect polymer coatings can now be produced; one method is to build up monolayers of 100 nm-diameter latex spheres under electrostatic attraction, discharge the layer and start again. Production of polymeric foam to the requisite specification, however, is still not possible to the necessary uniformity (or at least was not in 1980).

One of the rare publications to appear since 1980 that deal with ICF pellet (or 'target') production is a paper from Livermore National Laboratory describing the use of an array of gas microjets to levitate a pellet, to allow an ultrasmooth polymer surface layer to be made by plasma polymerization ${ }^{8}$.

One curious technique that is not discussed in Farnum and Fries's paper is the approach to making thin flanged micro-hemispherical shells by methods based on microelectronic technology. For instance, starting from circular etchwindows in a silicon surface, hemispherical depressions of high perfection can be etched; these are then boron-doped by diffusion into a thin surface layer and etched from the back with an etchant that does not attack boron-rich silicon ${ }^{9}$. It is a striking tribute to the micromechanical precision of modern silicon handling that the necessary smoothness and sphericity can be attained in this manner.

Others have recently urged the use of metallic glass microballoons and this is exciting much interest ${ }^{10,11}$. Such hollow microspheres can be formed by exploiting the instabilities of hollow liquid jets. Microballoons of a dense $\mathrm{Au}-\mathrm{Pb}-\mathrm{Sb}$ glass have been made with surface smoothness and sphericity of a very high order. How such balloons are to be incorporated in a complete target is not disclosed. Such metallic glasses are known to be highly permeable to hydrogen and thus should not be difficult to fill with fuel. More generally, Lee has very recently outlined a range of other uses for metallic microballoons made from unstable hollow jets, including a rocket fuel ${ }^{12}$. A new technology seems to be emerging.

It would seem that a new generic name is required for the congeries of techniques which have been developed to make ICF fuel pellets - 'materials microtechnology' perhaps? When the full account of this work is published, it will be seen as an essential part of the history of modern materials science and technology. But we shall have to await wholesale declassification before we can know how successful the ICF approach to nuclear fusion currently is. Meanwhile, the interested reader is referred to a concise textbook ${ }^{13}$ on thermonuclear fusion that discusses the essential characteristics of inertial confinement.

2. Nygren, R.E et al. (eds) J. Nucl. Mat. 103 (1981).

3. Conn, R.W. J. Nucl. Mat. 100, 139 (1981)

4. Wiffen, F.W. et al (eds) J. Nucl. Mat. 85 \& 86 (1979).

5. Farnum, E.H. \& Fries, R.J. Res Mechanica 10, 87 (1984).

6. Hendricks, C.D. J. Nucl. Mat. 85 \& 86, 79 (1979).

. Miller, J.R., Fries, R.J. \& Press W.J. J. Nucl. Mat. 85 \& 86, 121 (1979).

8. Crane, J.K et al. J. Vac, Sci. Technol, 20, 129 (1982).

Wise, K.D., et al. J. Nucl. Mat. 85 \& 86, 103 (1979).

10. Lee, M.C., Kendall, J.M. \& Johnson, W.L. Appl. Phys. Lett. 40, 382 (1982).

11. Johnson, W.L. \& Lee, M.C. J. Vac. Sci. Technol. A 1, 1568 (1983).

2. Lee, M.C. paper presented at Sth Tut. Conf. on Rapidly Quenched Metals, Würzburg, 3-7 Sept. 1984. To be published by North Holland.

13. Hagler, M.O. \& Kristiansen, M. An Introduction to Controlled Thermonuclear Fusion (Lexington Books, Lexington M.A. 1977)

Robert W. Cahn was formerly Professor of Materials Science at the Univesity of Sussex and Professor of Metallurgy at the University of Paris-South. He can be contacted at 6 Storey's Way, Cambridge CB3 ODT. 